\title{
Propuesta tipológica forestal para los bosques de Quercus pyrenaica de la comarca del Bierzo del noroeste de España, empleando análisis multivariante
}

\author{
Forest typology proposal for the Quercus pyrenaica in the Bierzo region, \\ northwest Spain, using multivariance analysis
}

\author{
Pablo Cruz ${ }^{\mathrm{a}}$, Alfonso Fernández ${ }^{\mathrm{b}}$, José A Reque ${ }^{\mathrm{c}}$ \\ Autor de correspondencia: aUniversidad Mayor, Camino La Pirámide 5750, Huechuraba, Santiago, Chile, tel.: 56-2-3281367, \\ pablo.cruzj@mayor.cl \\ bUniversidad de León, 24400 Ponferrada, España. \\ cUniversidad de Valladolid, Escuela Técnica Superior de Ingenierías Agrarias, 34071 Palencia, España.
}

\begin{abstract}
SUMMARY
The objective of this work is to propose a typology of Quercus pyrenaica in the district of Bierzo, northwest Spain, applying multivariance statistics to the plots of the third national forestry inventory. Six descriptor variables were selected and nine auxiliary calculated: the composition was represented with the cover value, and the age structure with forest calibers in 4 classes. Following this method, the typology was elaborated on the base of 15 variables. The stages of the statistical analyses were: factor analyses and factor determination with maximum explained variance, clusters analyses and dendrogram confection to decide the number of types and discrimination analyses to find the variables with main discriminating power. The variables set was considered appropriate for the confection of typology because of the statistics values achieved. Thus, six types that described adequately the conditions of pastures (grazing grounds) were determined, among them: pure pole stand, degraded pole stand and mixed forests, which have been proposed as frequent for the Community of Castilla y León as well. Furthermore, this methodology allowed establishing a dichotomous key to assign new situations to the proposed types, through the use of clearly differentiable numerical parameters as well as an easily observable on field. Considering the similitude between South American Nothofagus's forests and those of European fagaceous in which typologies have developed, they make of these a promising tool of forest management for Chile and Argentina.
\end{abstract}

Key words: Quercus pyrenaica, forest typology, multivariance statistics, national forestry inventory.

\section{RESUMEN}

El objetivo de este trabajo fue proponer una tipología para Quercus pyrenaica en la comarca del Bierzo, España, aplicando estadística multivariante a las parcelas del tercer Inventario Forestal Nacional (IFN3). Fueron seleccionadas seis variables descriptoras y calculadas otras nueve auxiliares; la composición se representó con el valor de cobertura y la estructura de edades con tamaños de los diámetros en cuatro clases. Así, esta tipología se elaboró sobre la base de 15 variables. Las etapas del análisis estadístico fueron análisis factorial y determinación de factores con máxima varianza explicada, confección de dendrograma para determinar el número de tipos, y análisis discriminante para hallar las variables con mayor poder de discriminación. El grupo de variables se consideró adecuado para la determinación de la tipología a la luz de los estadísticos logrados. Así, fueron encontrados seis tipos que describen adecuadamente las condiciones de dehesas, latizales puros o degradados y bosques mixtos que han sido propuestos con frecuencia para la Comunidad de Castilla y León. Esta metodología permitió además establecer una clave dicotómica para asignar nuevas situaciones a los tipos propuestos, mediante el uso de parámetros cuantitativos diferenciadores y de fácil observación en campo. Considerando las similitudes entre los bosques de Nothofagus spp. sudamericanos y los de fagáceas europeas en que se han desarrollado las tipologías, hacen de éstas una prometedora herramienta de gestión forestal para Chile y Argentina.

Palabras clave: Quercus pyrenaica, tipología forestal, estadística multivariante, inventario forestal nacional.

\section{INTRODUCCIÓN}

La clasificación de la vegetación, que nace en los primeros años del siglo XIX, se basó en la sociología vegetal y se sustentó en dos ejes fundamentales: los estudios florísticos y los fisionómicos (Acot 1990). La búsqueda de tipos vegetacionales fue un proceso rico en conceptua- lización de los ecosistemas mundiales, pero adoleció de estandarizaciones que facilitaran metodologías de aplicación simple para nuevas situaciones, debido principalmente a que tanto los listados florísticos como las descripciones fisionómicas fueron abordados por escuelas locales, cada uno desarrollando sus propias nomenclaturas, como fueron las de Zürich-Montpellier, Uppsala y de Chicago, entre 
otras (Acot 1990). En Europa, hasta el año 1960 las clasificaciones más usadas fueron las de Zürich-Montpellier $\mathrm{y}$ en específico los trabajos de Braun-Blanquet (Kent y Coker 1992). Después de esos años ha existido una considerable producción de trabajos de fitosociología para la península Ibérica (Rivas-Martínez 1976, Rivas-Martínez y Penas 2003).

En Chile en particular, la clasificación sobre la base de relaciones biogeográficas ha generado importantes avances; destacan las de Gajardo (1994) y de Luebert y Pliscoff (2006) a escala nacional. Por otra parte, la clasificación de tipos forestales (Donoso 1981) sigue vigente dado su acertado esfuerzo de síntesis de grupos vegetacionales tan heterogéneos. Trabajos como los citados han permitido esquematizar los ecosistemas forestales a escala nacional, teniendo como principal objetivo entregar descripciones de gran escala territorial. Los tipos forestales (Donoso 1981) permitieron generar normativas para los bosques de Chile y establecer límites generales respecto de la silvicultura que puede ser aplicada en cada tipo. Para ello, dichas normativas fueron construidas sobre una combinación de variables cualitativas descriptoras de los bosques y criterios político-administrativos.

En la evolución descrita, un nivel más específico ha sido la agrupación de bosques con el objetivo de sistematizar su gestión silvicultural, estableciéndose el concepto de tipología forestal (TF) como una síntesis sobre una terminología común que se construye a partir de características relevantes para definir los objetivos de gestión permanente, y más aún, para la proposición de actividades silviculturales para el presente.

Uno de los primeros trabajos con esta orientación es el de Herbert y Rebeirot (1985), en bosques de coníferas en el macizo del Jura en Francia, quienes logran establecer siete tipos, caracterizados silvícola y dendrométricamente, un diagrama de evolución natural y recomendaciones silviculturales para cada uno. Así también, se destaca el trabajo que Chauvin et al. (1994) presentaron respecto de la gestión de bosques de protección en Los Alpes franceses, para lo cual propusieron una tipología estructural de dichos bosques basada en estratificación vertical, el área basal y la microestructura. Si bien los objetivos de la confección de TF es la gestión de bosques, se destaca en los diversos trabajos posteriores la amplia gama de fuentes de información con la que ellas pueden ser construidas, tales como clasificación estereoscópica de fotografías aéreas (Bebi et al. 2001), parcelas temporales de inventario forestal (Rivera 2007), inventarios levantados especialmente con ese propósito (Reque et al. 2007) y, frecuentemente, sobre la base de inventarios nacionales (Serrada et al. 1993, Reque 2004, Roig et al. 2007).

La maduración del concepto de TF permite describir tres aspectos metodológicos comunes. Si bien es posible la utilización de caracterizaciones fitosociológicas basadas en variables cualitativas, la tendencia es dar preponderancia a variables cuantitativas, especialmente dasométricas (Gaudin
1997). Por otra parte, considerando que la confección de una tipología forestal es una forma de representación de bosques, en que muchas variables pueden ser continuas, resulta de importancia la generación de claves de determinación de cada tipo y, en consecuencia, una de las potencias de una TF radica en poder asignar los bosques a algún tipo sobre simples inspecciones visuales, resultando en ahorros por conceptos de diagnóstico (Gaudin 1997, Reque 2004). En tercer término, con la aparición de procesadores computacionales, por una parte, y el acopio de información sistematizada de la vegetación por otra, existe una tendencia creciente a emplear métodos numéricos en las clasificaciones, debido a su potencia para procesar y analizar casos con gran número de variables actuando en forma simultánea, además de mejorar la objetividad de los resultados y permitir, en consecuencia, tanto replicaciones como extrapolaciones posteriores (Kent y Coker 1992).

En este contexto, las herramientas derivadas de la estadística multivariante han sido profusamente usadas en la confección de TF (Bebi et al. 2001, Reque 2004, Roig et al. 2006, 2007, Tárrega et al. 2006, Aunós et al. 2007, Piqué et al. 2007, Reque et al. 2007, Gómez-Manzanedo et al. 2008), principalmente aquéllas que permiten definir grupos y analizar las variables que los originan. Los análisis frecuentemente aplicados son: análisis de agrupamiento, componentes principales, análisis factorial, análisis discriminantes y análisis de correlación canónica (McGarigal et al. 2000). A pesar del considerable grado de complejidad que pueden alcanzar estas herramientas, constituyen una potente alternativa de simplificación para efectos de confeccionar tipologías de cualquier índole, ya que permiten comprender las tendencias conjuntas e individuales de variables que afectan a una muestra, como es el caso de la vegetación $\mathrm{y}$ en particular los ecosistemas forestales.

Para bosques con predominancia de especies del orden Fagales, como los del mediterráneo europeo y los del sur del Cono Sur de América, la mayoría de los trabajos sobre TF se ha centrado en fagáceas. En Nothofagus spp. se puede mencionar el trabajo de Collado (2001), que propuso una tipología para los bosques de Tierra del Fuego, en que las especies preponderantes son N. pumilio (Poepp. et Endl.) Krasser, N. antarctica (G. Forst) Oerst y $N$. betuloides (Mirb.) Oerst. Un trabajo más específico es el publicado por Reque et al. (2007), quienes propusieron una caracterización silvícola y alternativas de gestión para bosques de $N$. antarctica de la provincia de Río Negro, Argentina.

Para Quercus spp., sin embargo, existen diversas propuestas de TF (Serrada et al. 1993, Vila y Díaz-Maroto 2002, Reque 2004, Piqué et al. 2007, Roig et al. 2007), de las cuales sólo dos se han realizado en específico para $Q$. pyrenaica Willd. (rebollo). Serrada et al. (1993) propusieron una tipología para rebollares en la Comunidad Autónoma de Madrid, basada en inventarios preexistentes para un ámbito territorial de 23 mil hectáreas. Roig et al. (2007), trabajando con los datos del Tercer Inventario Nacional 
Forestal de España (INF3), propusieron una tipología de la especie para toda la comunidad de Castilla y León, que comprende más de 700 mil hectáreas.

El rebollo en la Comunidad Autónoma de Castilla y León podría ser considerado la especie forestal más representativa debido a que ocupa el $24 \%$ de su superficie arbolada con 722.773 hectáreas. Además, los resultados del IFN3 indican que su superficie está aumentando en la región (Consejería de Medio Ambiente, Junta de Castilla y León 2005). La especie se distribuye por el norte de África (Marruecos), gran parte de la Península Ibérica (salvo El Levante) y el oeste y suroeste de Francia (Castroviejo 1990). En zonas de clima mediterráneo de la Península Ibérica se distribuye en áreas con precipitaciones anuales superiores a los $650 \mathrm{~mm}$, y al menos un mes en condiciones de aridez. Es una especie intolerante, de hoja marcescente con una excepcional capacidad de rebrotar, incluso luego de incendios (Ceballos y Fernández de Córdoba y De la Torre 1971). Ello ha posibilitado que ocupe grandes extensiones en formaciones de monte bajo o medio en áreas de silvopastoreo. Luis-Calabuig et al. (2001) presentan una cronología de alteración iniciada hace 2.000 años, en la que las alteraciones constantes fueron el fuego y el ramoneo del ganado.

Actualmente el rebollo se presenta en bosquetes heterogéneos de baja densidad y de superficies muy irregulares por una constante de fragmentación causada por la ganadería, la agricultura, la infraestructura rural y la extracción de leña, principalmente. En los bosques de montaña se pueden encontrar los mejores ejemplares con alturas de hasta 25 metros, creciendo en formaciones más densas. Son frecuentes las formaciones adehesadas de amplias copas y troncos robustos y bajos debido a los continuos trasmoches (Tárrega et al. 2006).

$\mathrm{Su}$ densidad es relativamente baja situándose en 500 árboles/ha como media, con una cobertura en torno al $60 \%$. A pesar que bosques secundarios, puros y de especies intolerantes como éste se caracterizan por su simpleza estructural, el rebollo en particular posee una amplia variación de estados de desarrollo, densidades de las masas y origen de los árboles (Roig et al. 2007), derivados de las acciones antrópicas descritas (Tárrega et al. 2006).

El objetivo de este trabajo es elaborar una tipología forestal usando metodologías de estadística multivariante, para rebollares de la comarca del Bierzo, provincia de León de la Comunidad Autónoma de Castilla y León en España. Se hace énfasis en los aspectos metodológicos y su potencia descriptora en atención a la utilidad que podrían tener estas técnicas para la caracterización silvícola de bosques templados similares a los de rebollo, como son los dominados por el género Nothofagus del Cono Sur de América.

\section{MÉTODOS}

Condiciones de sitio. El área de estudio correspondió a los bosques de rebollo de la comarca del Bierzo (figura 1), que presenta una orografía de hoya rodeada de montañas, cuyas alturas máximas bordean los $2.000 \mathrm{~m}$ s.n.m. Presenta un clima mediterráneo y atlántico con temperatura media anual de $13^{\circ} \mathrm{C}$, oscilación térmica entre 5 y $21^{\circ} \mathrm{C}$ y precipitaciones anuales en las zonas de montaña en un rango de 900 a $1.300 \mathrm{~mm}$ (De la Torre 1996). En estas condiciones se estima que el período de receso vegetativo alcanza unos seis meses, desde diciembre a mayo (AEMET 2008). En términos generales sus suelos se caracterizan por su baja potencialidad agrícola, que restringe su uso básicamente a la repoblación forestal y a la regeneración natural. Según la clasificación norteamericana de suelos, se encuentran fundamentalmente los órdenes Inceptisoles y Entisoles, que en las zonas de montaña, donde frecuentemente se encuentra el rebollo, están constituidos por una mezcla de elementos finos, cuarcitas y pizarras (Santos et al. 2005).

Preparación de la información. Se utilizó la información del IFN3 de España realizado el año 2004. Éste fue un proyecto de escala nacional que consideró la confección previa de un mapa forestal, sobre el que se realizó la definición de los diferentes ecosistemas o estratos a describir. El levantamiento de información se realizó a partir de un muestreo con diseño sistemático con arranque aleatorio y asignación

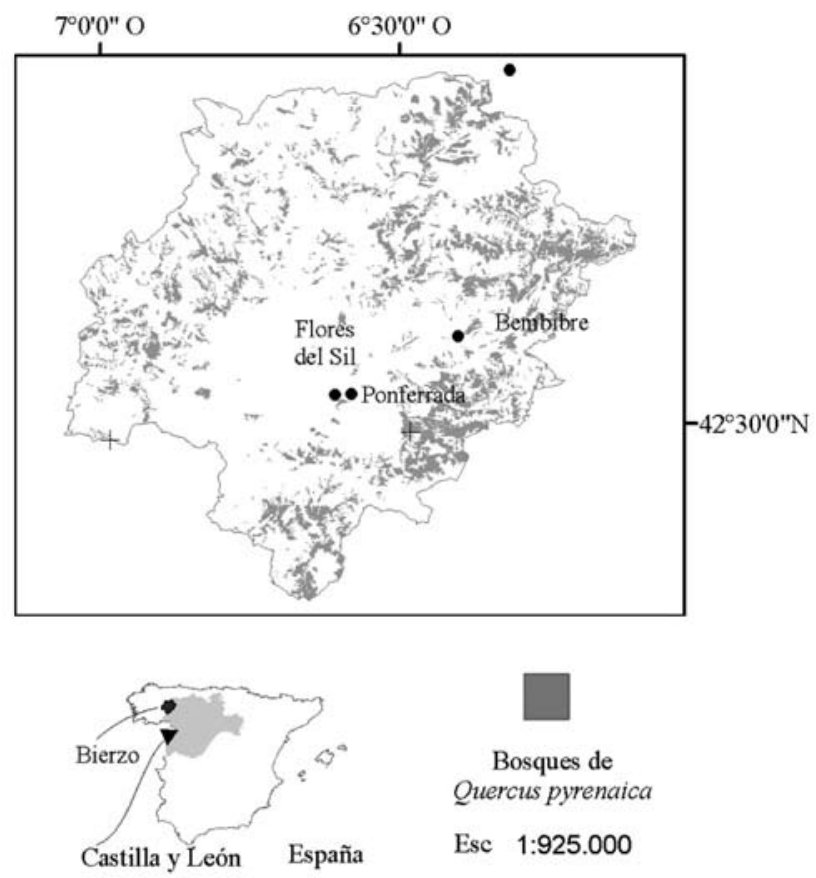

Figura 1. Mapa de localización de los bosques estudiados, en la comarca del Bierzo, de la provincia de León en la Comunidad Autónoma de Castilla y León.

Location map of the studied forests, in the district of Bierzo, province of León in the Autonomic Community of Castilla y León. 
proporcional a la superficie de cada estrato, del que resultó una densidad aproximada de un punto de muestreo por kilómetro cuadrado. Para la captura del conjunto de datos se estableció un sistema de parcelas circulares concéntricas que permitieron optimizar la colecta de datos. Así, la regeneración de especies arbóreas se registró en una parcela de radio $5 \mathrm{~m}$, el matorral leñoso en un radio de $10 \mathrm{~m}$, las especies arbóreas en $25 \mathrm{~m}$ y fenómenos erosivos en un radio de $60 \mathrm{~m}$. La información registrada en cada punto se clasificó en 12 bloques temáticos que describieron tanto condiciones físicas como de la vegetación. De la vegetación arbórea se registraron aspectos dasométricos, dendrométricos y otros cualitativos estructurales de la vegetación. Se pretende que el INF3 sea de carácter permanente, por lo que tanto el establecimiento de las parcelas como su procesamiento se realizan en forma dinámica en el tiempo y en directa relación con el Mapa Forestal Nacional. Los resultados del procesamiento de datos y análisis secundarios pueden ser consultados con el programa BASIFOR 2.0 (Bravo et al. 2002), el cual permite seleccionar atributos y variables, y calcular índices y funciones a partir de la información recogida para cada parcela.

Del IFN3 se usaron aquellas parcelas que cumplieron las condiciones de: a) pertenecer a la comarca del Bierzo (se incluyeron 36 parcelas adicionales fuera de la comarca), hasta una distancia de $35 \mathrm{~km}$ para aumentar el número de unidades muestrales (figura 1); b) representar bosques con predominancia de rebollo, condicionada a que la suma de la densidad relativa (densidad del rebollo sobre la densidad total de la parcela) y el área basal ( $\mathrm{AB}$ ) relativa ( $\mathrm{AB}$ del rebollo sobre el total del $\mathrm{AB}$ de la parcela) fuese igual o mayor a 100 (bosques puros de rebollo o mixtos en que la especie fuera importante). Así, fueron seleccionadas 148 parcelas, que en conjunto tuvieron una representación territorial de 53.100 hectáreas de bosques de rebollo dentro de la comarca.

Variables utilizadas. Fueron escogidas sólo variables originales de campo y sin procesamientos en el programa BASIFOR 2.0, descartándose volúmenes e índices, entre otras (cuadro 1). En la mayoría de las propuestas de tipologías, la estructura de edades del bosque se representa por clases diamétricas de troncos o fustes fino, medio, grueso y muy grueso (Bebi et al. 2001, Reque 2004, Roig et al. 2006, Reque et al. 2007). Para este estudio se definieron categorías a partir de la dispersión en percentil del diámetro cuadrático medio (Dg). Así, en cada parcela fue determinado el porcentaje de fustes en alguna de las categorías, según la frecuencia por clase diamétrica de la densidad y el $\mathrm{AB}$, generándose ocho nuevas variables (cuadro 1). Fue necesario adicionar una variable que permitiera identificar aquellas situaciones en que el rebollo se mezclaba con otras especies arbóreas. Se determinó un valor de cobertura, como la suma de los porcentajes de densidad y AB de la especie sobre el de todas las especies de cada parcela.

Cuadro 1. Variables originadas en los datos del IFN3 y variables auxiliares elaboradas para el análisis.

Variables originated in the data of the IFN3 and auxiliary variables elaborated for the analysis.

\begin{tabular}{|c|c|c|c|c|}
\hline & $\mathrm{N}^{\mathrm{o}}$ variable & Variable & Unidad & Descripción \\
\hline \multirow{6}{*}{ 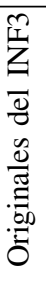 } & 1 & Densidad $(\mathrm{N})$ & (árboles/ha) & Número de árboles de la especie en cada parcela \\
\hline & 2 & Área basal (AB) & $\left(\mathrm{m}^{2} / \mathrm{ha}\right)$ & Área basimétrica del rebollo en cada parcela \\
\hline & 3 & Diámetro cuadrático medio $(\mathrm{Dg})$ & $\mathrm{cm}$ & Considerado sólo de rebollo \\
\hline & 4 & D. dominante (Do) & $\mathrm{cm}$ & \\
\hline & 5 & Altura media $(\mathrm{Hm})$ & $\mathrm{m}$ & \\
\hline & 6 & Altura dominante (Ho) & $\mathrm{m}$ & Considerado sólo de rebollo \\
\hline \multirow{15}{*}{ 总 } & & Fuste fino & - & Clases diamétricas Fustes Finos: 7,5, 10 y 15. \\
\hline & 7 & fus_np_f & $\%$ & $\mathrm{n}_{\text {clases finas }} / \mathrm{N}_{\text {total }}$ \\
\hline & 8 & fus gp_f & $\%$ & $\mathrm{ab}_{\text {clases finas }} / \mathrm{AB}_{\text {total }}$ \\
\hline & & Fuste medio & - & Clases diamétricas Fustes Medios: 20, 25 y 30. \\
\hline & 9 & fus_np_m & $\%$ & $\mathrm{n}_{\text {clases medias }} / \mathrm{N}_{\text {total }}$ \\
\hline & 10 & fus gp_m & $\%$ & $\mathrm{ab}_{\text {clases medias }} / \mathrm{AB}_{\text {total }}$ \\
\hline & & Fuste grueso & - & Clases diamétricas Fustes Gruesos: 35, 40 y 45. \\
\hline & 11 & fus_np_g & $\%$ & $\mathrm{n}_{\text {clases gruesas }} / \mathrm{N}_{\text {total }}$ \\
\hline & 12 & fus gp_g & $\%$ & $\mathrm{ab}_{\text {clases gruesas }} / \mathrm{AB}_{\text {total }}$ \\
\hline & & Fuste muy grueso & - & Clases diamétricas Fustes Muy gruesos $>45$. \\
\hline & 13 & fus_np_mg & $\%$ & $\mathrm{n}_{\text {clases mgruesas }} / \mathrm{N}_{\text {total }}$ \\
\hline & 14 & fus gp_mg & $\%$ & $\begin{array}{l}\mathrm{ab}_{\text {clases mgruesas }} / \mathrm{AB}_{\text {total }} \\
\text { Para todas las especies en cada parcela. }\end{array}$ \\
\hline & 15 & Valor de Cobertura (VC) densidad & - & NTP + ABTP, valor máximo 200 \\
\hline & & relativa $(\mathrm{NTP})$ & $\%$ & Densidad del rebollo sobre el total de la parcela. \\
\hline & & $\mathrm{AB}$ relativa $(\mathrm{ABTP})$ & $\%$ & $\mathrm{AB}$ del rebollo sobre el $\mathrm{AB}$ total de la parcela. \\
\hline
\end{tabular}


Modelo estadístico. El proceso estadístico tuvo como finalidad agrupar las parcelas de acuerdo a la similitud que presentaban respecto del conjunto de variables preestablecidas. Obtenido el agrupamiento, fue posible proponer a cada grupo como un componente de la tipología. El análisis fue realizado con el programa SPSS 15.0.

a) Análisis factorial (AF). Fue aplicado un AF sobre las 15 variables con el objetivo de reducir la dimensión de datos sin pérdida de información y verificar si el modelo de datos era pertinente. Para ello se analizó la matriz de correlaciones de las variables, a partir de su determinante como indicador del grado de las intercorrelaciones generales, la medida de adecuación de la muestra de Kaiser-MaeyerOlkin KMO como indicador del grado en que las variables pueden explicar la correlación entre pares de variables y la prueba de esfericidad de Bartlett como indicador de la existencia de correlaciones entre algunas de las variables (Martín et al. 1999). Luego se extrajo el número óptimo de factores mediante el método de componentes principales. En este proceso el primer factor resume la mayor cantidad de información del conjunto de datos, el segundo la restante y así sucesivamente hasta tener tantos factores como variables originales. Dado que el objetivo del proceso es reducir el número de variables sin perder información, fueron seleccionados sólo aquellos factores que aportaron al modelo un nivel de información significativa, mediante la determinación de valores propios (o autovalores) que pueden interpretarse como la cantidad de varianza explicada por cada factor, aceptándose aquellos con autovalor mayor a 1, y que representa la cantidad de veces que un autovalor debe ser mayor que el autovalor promedio de la matriz (regla de Kaiser) (Pardo y Ruiz 2005). La varianza total explicada corresponde a la suma de la varianza aportada por cada factor. Como la extracción de algunos factores del modelo hace que la explicación de la varianza de las variables originales no sea completa (o 100\%), se determinó su comunalidad (proporción de la varianza explicada por los factores de cada una de las variables) en una matriz de componentes después de la extracción. Para una mejor interpretación de la relación de las variables originales y los factores, se aplicó una rotación ortogonal a la matriz de componentes, mediante una combinación lineal de la primera que permite ayudar a interpretar los casos cuando las cargas factoriales no están claras. El método usado fue varimax para minimizar el número de variables que tienen saturaciones altas en un factor y así mejorar la interpretación de los resultados (Martín et al. 1999, Pardo y Ruiz 2005). Finalmente se determinaron las puntuaciones de cada parcela de inventario en cada factor del modelo y se adicionaron como una nueva variable a los datos para la clasificación de tipos.

b) Clasificación en tipos (CT). Se realizó un análisis de agrupamientos con el método de varianzas mínimas (Ward), usando como índice de similitud las distancias euclidianas al cuadrado, permitiendo todos los posibles grupos, usando como variables las puntuaciones factoriales obtenidas para cada parcela. Con este resultado se construyó un dendrograma que permitió observar a qué distancia estandarizada cada grupo es considerado semejante con otra parcela o grupo. La altura de las líneas verticales representa la distancia a que se unen primero las parcelas y luego los grupos, medida por la suma de los cuadrados del error. El número de grupos definitivos fue obtenido trazando una línea de corte horizontal de las ramas del árbol donde las distancias de agrupamiento cambian bruscamente, para hacerse significativamente largas (Pardo y Ruiz 2005, Martín et al. 1999).

c) Análisis discriminante (AD). Mediante un $\mathrm{AD}$ se determinó qué variables permitieron diferenciar mejor a los grupos, el que consistió en construir una combinación lineal (función discriminante) con las variables independientes que predijo la pertenencia de una parcela (nueva o de esta muestra) a los tipos determinados, usando la asignación original como variable dependiente (modelo de Fisher, estadígrafo Lambda de Wilks y valor $\mathrm{F}$ para determinar las condiciones de incorporación de las variables (Pardo y Ruiz 2005)). A partir de las funciones de clasificación de Fisher se reasignaron las parcelas a los grupos y luego se comparó esta segunda asignación con la obtenida originalmente, para determinar un nivel porcentual de agrupamiento. Además, el modelo lineal de Fisher permitió jerarquizar las variables respecto de su contribución al agrupamiento para facilitar la creación de una guía de tipificación con los resultados expuestos.

Tipificación estructural y guía de clasificación. La tipificación estructural fue, para este caso, un procedimiento por el cual se buscó un método de reconocer los tipos encontrados sobre la base de las diferencias en las variables usadas. El proceso terminó con la confección de una guía de clasificación como herramienta práctica para la gestión de bosques, a partir de la cual sería posible describir un rodal cualquiera de la comarca del Bierzo, basándose en las características de los tipos propuestos. Se construyó sobre las diferencias que mostraron las variables definidas como de alto valor discriminatorio entre tipos, determinándose los límites de confianza del conjunto de variables al $95 \%$. La descripción gráfica del mencionado procedimiento se representó en un flujo en que las opciones de avance son dicotómicas y definidas por los límites de confianza estimados para las variables. Dicho flujo, además, constituyó la guía de clasificación que se propone en este estudio.

\section{RESULTADOS}

Análisis estadístico. En el AF el grado de correlación entre las variables originales (determinante de la matriz 
Cuadro 2. Análisis de la varianza del modelo factorial.

Variance analysis of factorial model.

\begin{tabular}{|c|c|c|c|c|c|c|c|c|c|}
\hline \multirow{3}{*}{$\begin{array}{c}\text { Componente } \\
\text { (factor) }\end{array}$} & \multirow{2}{*}{\multicolumn{3}{|c|}{ Autovalores iniciales }} & \multicolumn{6}{|c|}{ Sumas de las saturaciones al cuadrado } \\
\hline & & & & \multicolumn{3}{|c|}{ de la extracción } & \multicolumn{3}{|c|}{ de la rotación } \\
\hline & Total & $\begin{array}{c}\% \text { de la } \\
\text { varianza }\end{array}$ & $\begin{array}{c}\% \\
\text { acumulado }\end{array}$ & Total & $\begin{array}{c}\% \text { de la } \\
\text { varianza }\end{array}$ & $\begin{array}{c}\% \\
\text { acumulado }\end{array}$ & Total & $\begin{array}{c}\% \text { de la } \\
\text { varianza }\end{array}$ & $\begin{array}{c}\% \\
\text { acumulado }\end{array}$ \\
\hline 1 & 6,19 & 41,27 & 41,27 & 6,19 & 41,27 & 41,27 & 3,98 & 26,57 & 26,57 \\
\hline 2 & 2,33 & 15,55 & 56,82 & 2,33 & 15,55 & 56,82 & 3,145 & 20,96 & 47,54 \\
\hline 3 & 2,17 & 14,48 & 71,30 & 2,17 & 14,48 & 71,30 & 3,030 & 20,19 & 67,74 \\
\hline 4 & 1,81 & 12,07 & 83,38 & 1,81 & 12,07 & 83,38 & 2,256 & 15,03 & 82,77 \\
\hline 5 & 1,09 & 7,31 & 90,69 & 1,09 & 7,31 & 90,69 & 1,188 & 7,91 & 90,69 \\
\hline $6 y+$ & $<$ a 1,0 & - & - & - & - & - & - & - & - \\
\hline
\end{tabular}

de correlaciones) resultó cercano a cero, indicando una alta correlación entre las variables del modelo. Se aceptó además la posibilidad de explicar correlaciones de pares de variables por otras, al obtenerse un estadístico de KMO mayor a 0,6 (igual a 0,604). Así también, la significancia en la prueba de esfericidad de Bartlett, como indicador de que una o más variables pudieran ser representadas como una combinación lineal de otras, arrojó valores menores a 0,05 (0 con 105 grados de libertad), establecido como satisfactorio (Pardo y Ruiz 2005). Cinco factores alcanzaron autovalores mayores a 1 (cuadro 2), que en conjunto sumaron una varianza mayor al $90 \%$, y que pueden ser considerados suficientes para generar un agrupamiento (Martín et al. 1999).

En la matriz de componentes rotados se presenta el peso o la carga de cada variable en los factores (cuadro 3), obtenidos con el método de componentes principales y rotación varimax (se han eliminado las cargas entre $-0,3$ y 0,3 para simplificar el análisis), comprobándose que la mayoría satura en algún factor. El primer factor refleja los porcentajes de fustes de tamaño medio y fino (clases de DAP $<15 \mathrm{~cm}$ ) tanto en densidad como en AB; el segundo factor refleja el porcentaje de fustes muy gruesos en densidad y $\mathrm{AB}$, y el diámetro cuadrático medio y el diámetro dominante; el tercer factor refleja los fustes gruesos en densidad y $\mathrm{AB}$, el cuarto factor la densidad y el $\mathrm{AB}$, y el quinto factor el valor de cobertura. La confección del dendrograma permitió determinar seis tipos (figura 2).

La aplicación combinada del estadístico lambda Wilks para seleccionar las variables y el estadístico $\mathrm{F}$ como criterio de evaluación como poder de discriminación del modelo efectuada en el $\mathrm{AD}$, determinó que las variables con mayor poder de discriminación fueron cinco de las quince (en orden de importancia fus_np_mg, Vc, fus_gp_f, N y fus_gp_g). Los valores de lambda Wilks para las funciones del modelo fueron relativamente bajos, indicando un bajo solapamiento de los grupos, y su nivel de significancia Chi-cuadrado arrojó una significancia de 0,0 (cuadros 4 y 5). La misma muestra reclasificada por el modelo arrojó una concordancia de $93,2 \%$ respecto de la clasificación
Cuadro 3. Matriz de componentes rotados.

Rotated components matrix.

\begin{tabular}{lccccc}
\hline \multirow{2}{*}{ Variables } & \multicolumn{5}{c}{ Componentes } \\
\cline { 2 - 6 } & 1 & 2 & 3 & 4 & 5 \\
\hline Vc & - & - & - & - & 0,93 \\
$\mathrm{~N}$ & - & - & - & 0,83 & - \\
$\mathrm{Dg}$ & 0,39 & 0,78 & 0,45 & - & - \\
$\mathrm{Do}$ & 0,38 & 0,72 & 0,42 & 0,33 & - \\
$\mathrm{AB}$ & - & - & - & 0,93 & - \\
Hm & 0,51 & - & 0,46 & 0,35 & $-0,32$ \\
Ho & 0,37 & - & 0,36 & 0,66 & $-0,33$ \\
fus_np_g & - & - & 0,97 & - & - \\
fus_gp_m & 0,96 & - & - & - & - \\
fus_np_f & $-0,80$ & - & $-0,47$ & - & - \\
fus_np_m & 0,98 & - & - & - & - \\
fus_np_mg & - & 0,97 & - & - & - \\
fus_gp_f & $-0,81$ & - & $-0,42$ & - & - \\
fus_gp_g & - & - & 0,95 & - & - \\
fus_gp_mg & - & 0,90 & - & - & - \\
\hline
\end{tabular}

Vc: valor de cobertura; $\mathrm{N}$ : densidad; Dg: diámetro cuadrático medio; Do: diámetro dominante; $\mathrm{AB}$ : área basal; Hm: altura media; Ho: altura dominante; fus_np_f, m, g y mg: porcentaje de fustes (en densidad) de tamaños finos, medios, gruesos y muy gruesos; fus_gp_f, m, g y mg: porcentaje de fustes (en $\mathrm{AB}$ ) de tamaños finos, medios, gruesos y muy gruesos.

original. Las parcelas mal clasificadas fueron reasignadas extrayéndose de su tipo original y asignándose al nuevo tipo pronosticado, lo que determinó $97 \%$ de parcelas correctamente clasificadas.

Tipología estructural. El análisis estadístico multivariante finalmente permitió proponer seis tipos. Las claves de clasificación tipológica resultante se presentan en la figura 3. Las diferencias en los valores que tomó cada variable discriminatoria en cada tipo fueron evidentes y sólo bastó contrastar los límites de confianza en las variables escogidas 


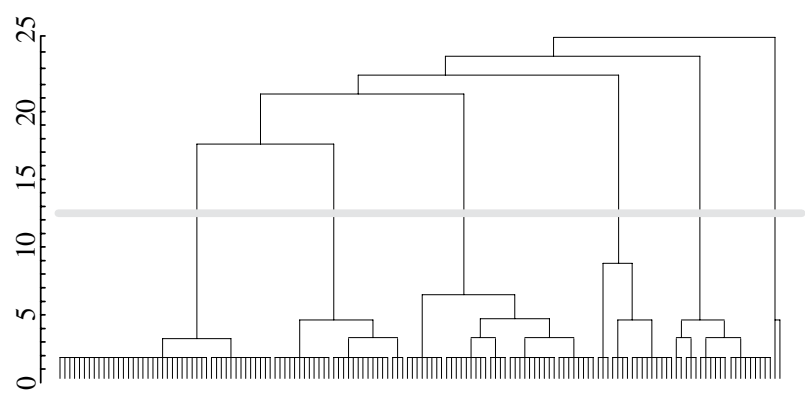

Figura 2. Dendrograma de agrupamiento. En el eje de las abscisas, cada línea corresponde a alguna de las parcelas del INF3 consideradas. El eje de las ordenadas representa las distancias de aglomeración (métodos de aglomeración de Ward), reescaladas. La línea gris representa el punto de corte para la formación de grupos.

Grouped dendrogram. At the $\mathrm{x}$-axis, each line corresponds to each one of the plots of INF3 considered. The y-axis represents the distances of crowd (Ward's Methods of crowd), rescaled. The gray line shows point of cut by group formation.

Cuadro 4. Estadísticos lambda de Wilks global para el modelo generado en cada paso.

Global lambda Wilks statistics, for model generated in each step.

\begin{tabular}{ccccc}
\hline $\begin{array}{c}\text { Contraste de las } \\
\text { funciones }\end{array}$ & $\begin{array}{c}\text { Lambda de } \\
\text { Wilks }\end{array}$ & Chi-cuadrado & gl & Sig. \\
\hline 1 a la 5 & 0,001 & 1021,271 & 30 & 0,000 \\
2 a la 5 & 0,007 & 708,388 & 20 & 0,000 \\
3 a la 5 & 0,039 & 457,115 & 12 & 0,000 \\
4 a la 5 & 0,155 & 262,920 & 6 & 0,000 \\
5 & 0,436 & 117,198 & 2 & 0,000 \\
\hline
\end{tabular}

gl: grados de libertad; Sig: significancia.

Cuadro 5. Coeficientes estandarizados de las funciones discriminantes canónicas.

Standardized coefficients of the canonical discrimination functions.

\begin{tabular}{lrrrrr}
\hline \multirow{2}{*}{ Variables } & \multicolumn{5}{c}{ Función } \\
\cline { 2 - 6 } & \multicolumn{1}{c}{1} & \multicolumn{1}{c}{2} & \multicolumn{1}{c}{3} & \multicolumn{1}{c}{4} & \multicolumn{1}{c}{5} \\
\hline VC & $-0,491$ & 0,894 & 0,002 & $-0,023$ & $-0,117$ \\
N & 0,260 & $-0,217$ & $-0,392$ & 0,841 & $-0,802$ \\
AB & $-0,369$ & 0,238 & 0,561 & 0,206 & 0,700 \\
fus_np_mg & 0,953 & 0,355 & $-0,090$ & 0,105 & 0,088 \\
fus_gp_f & $-0,173$ & 0,022 & $-0,411$ & 0,252 & 1,094 \\
fus_gp_g & $-0,076$ & 0,000 & 0,652 & 0,490 & 0,746 \\
\hline
\end{tabular}

para identificar los tipos. En el cuadro 6 se presenta una síntesis en que se generalizan las variables para ayudar a la caracterización de cada tipo. a) Tipo latizal bajo de baja densidad (T1). Corresponden a bosques de aspecto juvenil, acompañados ocasionalmente por algunos árboles de diámetros mayores en estratos superiores, pero en los que predominan notoriamente las clases diametrales pequeñas. Por esta condición su aspecto es claramente de latizal bajo regular. A pesar que en este tipo se han agrupado bosques de diversas densidades, la máxima densidad definida es en torno a 415 árboles por hectárea (cuadro 6). Las situaciones de bosques menos densos de este grupo podrían representar las constantes de alteración que soportan los rebollares (Luis-Calabuig et al. 2001), además de sugerir que su mayoría han sido originados de monte bajo. En este tipo se agrupó el mayor porcentaje de parcelas con un $30 \%$ del total.

b) Tipo latizal alto (T2). Este tipo constituye un conjunto de alta homogeneidad en su dispersión diamétrica, lo que otorga una fisonomía de bosque. Esta fisonomía se refleja también en los tamaños predominantes en el tipo, principalmente de fustes de tamaño fino y medio. Las condiciones de densidad incluidas en él son similares a las del tipo 1, tanto en su número como en el rango de dispersión (rango en torno a 160 árboles por hectárea) (cuadro 6). Este tipo está representado en las parcelas del INF3 con un $27 \%$.

c) Tipo latizal bajo de alta densidad (T3). Poseen una fisonomía similar a la del tipo T1, en cuanto a su condición de desarrollo y la presencia ocasional de árboles en estratos superiores. La principal diferencia radica en las densidades que, en este caso, son las mayores encontradas respecto de esta clasificación y que se representan también en el mayor promedio de $\mathrm{AB}$ entre todos los tipos. Esta condición representaría bosques sin manejo o con bajos niveles de alteraciones desde su establecimiento; su alta densidad (media de 1.400 árboles/ha) podría considerarse como en sobredensidad si se compara con lo descrito por otros autores para la especie en tal estado de desarrollo (Madrigal et al. 2000, Carvalho et al. 2005). El T3 representa un $16 \%$ de las parcelas del INF3.

d) Tipo fustal joven (T4). Corresponde a bosques en que los árboles presentan claramente un desarrollo fustal con diámetros menores en torno a $\operatorname{los} 25 \mathrm{~cm}$, aunque en algunos casos puedan estar acompañados por individuos de menor talla, lo que es sugerido por la presencia, aunque escasa, de fustes finos. En esta condición, podrían presentarse variados tipos de estructuras, en parcelas regulares a otras más bien irregulares, pero en todos los casos sería característica la presencia mayoritaria de individuos en condición de fustal joven. Este tipo agrupa sólo un 10\% de las parcelas.

e) Tipo de bosques mixtos (T5). Corresponde a bosques con predominancia de rebollo y la presencia de otras especies arbóreas. La presencia de varios tamaños 


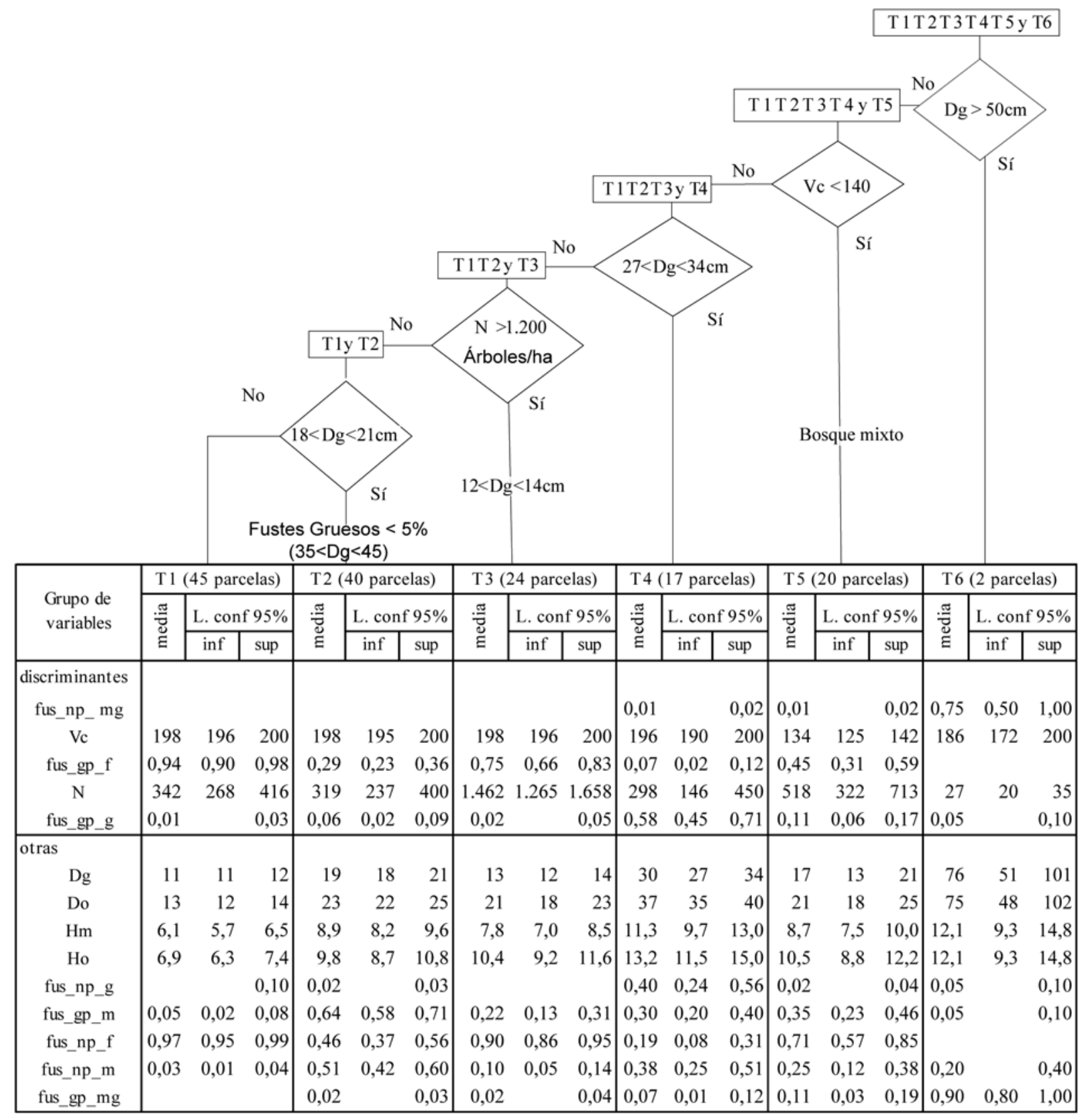

Figura 3. Clasificación a partir de las variables originales, según su potencia de discriminación. En el diagrama se han agregado algunas variables útiles para la identificación. L. conf 95\%: límites de confianza; inf: inferior; sup: superior.

Classification as from the original variables, according to his discriminating potency. In the diagram some useful variables have been added for the identification. L. conf $95 \%$ : confidence limits. inf: less. sup: higher.

de diámetros de los fustes (cuadro 6) determina que su fisonomía pueda en algunos casos presentarse como irregular. Las especies acompañantes más frecuentes son Betula sp., Quercus ilex L., Castanea sativa Mill. (castaño) y Pinus sylvestris L. (pino), que en ninguno de los casos se presentaron simultáneamente. Las dos primeras asociaciones podrían reflejar áreas ecotonales en los bordes de la distribución local de la especie, ya que en la zona Betula sp. se encuentra preferentemente a mayor altitud y Q. ilex en laderas de solana (exposición sur). Las otras dos podrían representar antiguas áreas de distribución natural de la especie que han sido transformadas en los actuales castañares y pinares de la comarca, en los que la especie persiste aún. Además de las descritas, el rebollo se encuentra asociado a un grupo de 15 especies arbóreas de manera ocasional, según la información contenida en las 20 parcelas que compusieron el tipo. Estos bosques representaron el $13 \%$ del total de parcelas. 
Cuadro 6. Síntesis de los atributos por cada tipo propuesto.

Synthesis of attributes for each proposed type.

\begin{tabular}{|c|c|c|c|c|c|c|}
\hline \multirow{2}{*}{ Atributo } & \multicolumn{6}{|c|}{ Bosque Tipo } \\
\hline & T 1 & $\mathrm{~T} 2$ & T 3 & $\mathrm{~T} 4$ & T 5 & T 6 \\
\hline Composición & puro & puro & puro & puro & mixto & puro \\
\hline Estados de desarrollo* & latizal bajo & latizal alto & latizal bajo & fustal joven & $\begin{array}{c}\text { latizal } \\
\text { alto y bajo }\end{array}$ & $\begin{array}{c}\text { adulto y sobre } \\
\text { maduro }\end{array}$ \\
\hline Densidad & baja & baja & alta & variable & variable & muy baja \\
\hline árboles/ha & 270 a 415 & 320 a 400 & 1.260 a 1.660 & 146 a 450 & 320 a 710 & 20 a 35 \\
\hline $\operatorname{Dg}(\mathrm{cm})$ & 11 a 12 & 18 a 21 & 12 a 14 & 25 a 35 & 13 a 21 & 51 a 101 \\
\hline Estructura de edades** & regular & regular amplia & regular & variable & variable & regular \\
\hline \multicolumn{7}{|l|}{ Clases diamétricas } \\
\hline \multicolumn{7}{|l|}{ finas $(\mathrm{DAP}<15 \mathrm{~cm})$} \\
\hline$\% \mathrm{~N}$ & 90 al 100 & 35 a 60 & 85 a 95 & 10 a 30 & 60 a 85 & no existen \\
\hline$\% \mathrm{AB}$ & 90 a 100 & 25 a 35 & 65 a 85 & 5 a 10 & 30 a 60 & no existen \\
\hline \multicolumn{7}{|c|}{ medias $(\mathrm{DAP}=20$ a $30 \mathrm{~cm})$} \\
\hline$\% \mathrm{~N}$ & no existen & 40 a 60 & 5 a 15 & 25 a 50 & 10 a 40 & 0 a 40 \\
\hline$\% \mathrm{AB}$ & no existen & 60 a 70 & 15 a 30 & 20 a 40 & 25 a 45 & 0 a 10 \\
\hline \multicolumn{7}{|c|}{ gruesas $(\mathrm{DAP}=35 \mathrm{a} 45 \mathrm{~cm})$} \\
\hline$\% \mathrm{~N}$ & 0 al 10 & no existen & no existen & 25 a 55 & 0 a 5 & 0 a 10 \\
\hline$\% \mathrm{AB}$ & 0 al 5 & no existen & 0 a 20 & 45 a 70 & 5 a 15 & 0 a 10 \\
\hline \multicolumn{7}{|c|}{ muy gruesas $(\mathrm{DAP}>45 \mathrm{~cm})$} \\
\hline$\% \mathrm{~N}$ & no existen & no existen & no existen & 0 a 5 & 0 a 5 & 50 a 100 \\
\hline$\% \mathrm{AB}$ & no existen & 0 a 5 & 0 a 5 & 0 a 10 & 5 a 20 & 80 a 100 \\
\hline
\end{tabular}

*Según Vita (1996). **Según la existencia de diferentes tamaños de fustes en cada tipo.

f) Tipo fustales adultos de baja densidad (T6). Son bosques de fisonomía claramente adulta y sobremadura en que los diámetros se encuentran en torno a los $70 \mathrm{~cm}$, superando en ocasiones un metro, con distanciamientos mayores a los $20 \mathrm{~m}$ entre individuos. La inexistencia de fustes de tamaño menor indicaría la ausencia de regeneración o estados juveniles. Este tipo podría representar dehesas tradicionales de la zona y podrían deberse a lo que Luis-Calabuig et al. (2001) indican como abandono de dehesas debido a la recesión de la ganadería en el noroeste del país, aunque su representación en el área sería extremadamente escasa, ya que en conjunto sólo llegaron al $1 \%$ de las parcelas.

\section{DISCUSIÓN}

La varianza obtenida en el AF fue mayor a las encontradas en trabajos de clasificación con estos métodos. Reque (2004), analizando 20 variables para su clasificación de Quercus petrea (Mattuschka) Liebl, logró un 85\% de explicación de la varianza en seis factores y Aunós et al. (2007), con sólo siete variables, llegaron a $72 \%$ con tres factores. Así también, el tipo de variables identificadas como relevantes para el agrupamiento es similar a aquellas encontradas por diversos autores. En los trabajos de Roig et al. (2006, 2007), para Juniperus thurifera L. y rebollos, respectivamente, se encuentra coincidencia en que espesura, distribución diamétrica y composición específica son las variables principales de agrupamiento. Las mismas variables cita Reque et al. (2007) para la separación de grupos en Nothofagus antarctica del norte de la Patagonia argentina, mientras que Reque (2004) señala que el calibre de fustes gruesos y su expresión en la densidad y AB fue determinante en $Q$. petrea. Aunós et al. (2007), en Abies alba Lindl., señalan como principales espesura, $\mathrm{AB}$ y estructura vertical. Tanto en este estudio como en los casos citados las variables de tipo dasométrico fueron indispensables.

Es una potente ventaja del método la depuración de variables principales en un gran grupo de datos, considerando que cada asociación forestal acentuará características propias y no necesariamente idénticas a otras. La clasificación de los árboles según su tamaño diametral ofrece buenos resultados para discriminar. Sin embargo, como la distribución diamétrica es una característica inherente 
a cada muestra, no es posible estandarizar los rangos de división, como método general. En efecto, cada estudio define tanto el número de categorías como sus límites; Reque (2004), Roig et al. (2006, 2007) y Aunós et al. (2007) definieron rangos diferentes para cada clase.

El agrupamiento de las parcelas sobre la base de análisis multivariante permitió establecer una clasificación dicotómica en la que los umbrales para la separación son significativamente claros y de tipo numérico. Ello permite basar las diferencias en atributos cuantitativos fácilmente reconocibles con una inspección visual del bosque.

Los tipos propuestos son similares a los encontrados en los trabajos de tipificación en rebollo realizado por Roig et al. (2007) para la vasta zona de la comunidad de Castilla y León y la comunidad de Madrid en conjunto, situación previsible considerando que la estructura del bosque de rebollo en la mayoría de los casos podrá describirse con las etapas de bosques coetáneos. Un aspecto relevante, sin embargo, es la diferencia respecto de la frecuencia en cada tipo y que permiten identificar las particularidades de este bosque en la comarca estudiada. Así, es destacable que para ambas comunidades las dehesas se representen en un $22 \%$, mientras que en el Bierzo en particular sean prácticamente inexistentes (1\%). Los latizales que los autores mencionados presentan como degradados son representados sólo con $22 \%$, que si se consideran homologables al T1, en el Bierzo serían mucho más abundantes (30\%). Si bien la similitud entre los latizales densos de Roig et al. (2007) y el T3 es especialmente alta (AB en torno a $25 \mathrm{~m}^{2} /$ ha y DAP medio de $13 \mathrm{~cm}$, según estos autores), su representación sería bastante menor (22\% para ambas comunidades y sólo $16 \%$ en el Bierzo). Los tipos fustales y bosques mixtos, en cambio, tienen una presencia relativamente similar.

La clasificación propuesta por Serrada et al. (1993), que define los tipos como de monte bajo, monte alto, degradados y dehesas, y que hace compleja una comparación con la propuesta en este trabajo, presenta, no obstante, una alta presencia de dehesas en la comunidad de Madrid (32\%) y una baja representación de bosques degradados (8\%), ambas condiciones en extremo diferentes en la zona estudiada.

Es interesante destacar la potencia del método para sintetizar eficientemente bosques que ocupan una considerable extensión del territorio, si se toma en cuenta que el trabajo de Roig et al. (2007) para Castilla y León podría abarcar 700 mil hectáreas de rebollares descritas por la Consejería de Medio Ambiente, Junta de Castilla y León (2005); y el de Serrada et al. (1993), unas 23 mil hectáreas de rebollares de la Comunidad Autónoma de Madrid.

Las ventajas metodológicas de tipologías forestales sobre la base de aplicación de estadística multivariante a inventarios dasométricos podrían ser de gran ayuda para mejorar la gestión de la vasta superficie de bosques templados del Cono Sur de Sudamérica. En efecto, los ecosistemas forestales con características similares a las descritas alcanzan una superficie cercana a los 9,5 millones de hectáreas, de los que existe una clasificación a gran escala, tanto en Chile como en Argentina (CONAF/CONAMA-BIRF 1999, Ministerio de Salud y Ambiente 2005). Las notables similitudes entre los bosques de fagáceas del hemisferio norte y los extensos bosques dominados por Nothofagus spp. del Cono Sur de Sudamérica en particular, se comprueban al comparar los tipos encontrados para rebollo en este estudio y aquellos descritos por Reque et al. (2007) para $N$. antarctica de la Patagonia argentina, específicamente respecto de la estructura que presentan aquellos, el tipo de variables que fueron definidas para la discriminación de los tipos y la heterogeneidad de los bosques como respuesta a las acciones antrópicas que los han afectado. En el mismo sentido se encuentran destacables coincidencias entre la dinámica estructural de haya (Fagus sylvatica L.) al norte de la Península Ibérica, contenida en la tipología propuesta por Gómez-Manzanedo et al. (2008), y N. pumilio en su distribución sur de Sudamérica respecto de su estructura, dinámica, densidades y estados de desarrollo (Donoso 1993, Cruz et al. 2005). Sin embargo, se hace fundamental en ambos territorios lograr avances sustantivos en la generación de inventarios de carácter nacional para disponer de datos homogéneos para dicho territorio.

\section{CONCLUSIONES}

La tipología conseguida refleja los estados frecuentes en que se presentan los bosques de rebollo de la comarca, divididos principalmente en bosques jóvenes, con diferentes grados de alteración y áreas en que la especie se presenta asociada a otras arbóreas. Así también, en comparación con las tipologías propuestas por otros autores, es posible indicar que en el Bierzo las dehesas serían casi inexistentes y que la frecuencia de bosques alterados o degradados sería mayor en comparación con los bosques de la especie en las comunidades de Castilla y León y de Madrid. Considerando la bondad de resultados que ofrece la combinación inventario forestal nacional-estadística multivariante para conceptualizar los bosques en escalas territoriales de grandes dimensiones, sería recomendable intentar relacionar inventarios como el usado con catastros o mapas de vegetación mediante este tipo de trabajos. Así, no sólo se obtendría la síntesis de situaciones para los tipos de bosque, sino que además el peso territorial de cada condición, y en consecuencia un valioso diagnóstico del estado del arte de los ecosistemas forestales regionales.

\section{REFERENCIAS}

Acot P. 1990. Historia de la Ecología. París, Francia. Alfaguara. $271 \mathrm{p}$.

AEMET (Agencia Estatal de Meteorología, ES). 2008. Datos climatológicos. Umbrales por provincia. Consultado 6 ago. 2008. Disponible en http://www.aemet.es/es/elclima/ datosclimatologicos/umbrales_provincia. 
Aunós A, E Martínez, R Blanco. 2007. Tipología selvícola para los abetos españoles de Abies alba Mill. Investigación Agraria 16 (1): 52-64.

Bebi P, F Kienast, W Schoënenberger. 2001. Assessing structures in mountain forests as a basis for investigating the forests' dynamics and protective function. Forest Ecology and Management 145: 3-14.

Bravo F, M Del Río, C Del Peso. 2002. El Inventario Nacional Forestal. Elemento clave para la gestión forestal sostenible. Valladolid, España. Fundación general de la Universidad de Valladolid. $191 \mathrm{p}$.

Carvalho J. 2005. O carvalho negral. Vila Real, Portugal. UTAD CEGE. 206 p.

Chauvin C, J Renaud, C Rupé. 1994. Stabilité et gestion des forets de protection. ONF-Bulletin technique (27): 37-52.

Castroviejo S. 1990. Platanaceae-Plumbaginaceae (partim). Flora ibérica: plantas vasculares de la Península Ibérica e Islas Baleares, Vol. 2. Madrid, España. Castroviejo Madrid. $952 \mathrm{p}$.

Ceballos y Fernández de Córdoba L, J De la Torre. 1971. Árboles y arbustos de la España peninsular. Madrid, España. Escuela Técnica Superior de Ingenieros de Montes. $321 \mathrm{p}$.

Collado L. 2001. Los bosques de Tierra del Fuego. Análisis de su estratificación mediante imágenes satelitales para el inventario forestal de la provincia. Multequina (10): 1-16.

CONAF/CONAMA-BIRF (Corporación Nacional Forestal/ Comisión Nacional de Medio Ambiente-Banco Internacional de Reconstrucción y Fomento, CL). 1999. Catastro y evaluación de los recursos vegetacionales nativos de Chile. Santiago, Chile. CONAF/CONAMA-BIRF. 89 p.

Consejería de Medio Ambiente, Junta de Castilla y León. 2005. Castilla y León crece con el bosque. León, España. Consejería de Medio Ambiente, Junta de Castilla y León. 48 p.

Cruz P, P Honeyman, C Caballero. 2005. Propuesta metodológica de ordenación forestal, aplicación a bosques de lenga de la XI Región. Bosque 2 (26): 57-70.

De la Torre J. 1996. Mapa forestal de España. Ponferrada. Hoja 3-3. Madrid, España. Ministerio de Medio Ambiente. 325 p.

Donoso C. 1981. Tipos Forestales de los bosques nativos de Chile. Doc de trabajo $N^{\circ}$ 38. Santiago, Chile. CONAFPNUD-FAO. $82 \mathrm{p}$.

Donoso C. 1993. Bosques templados de Chile y Argentina. Variación estructura y dinámica. Santiago, Chile. Universitaria. $483 \mathrm{p}$.

Gajardo R. 1994. La vegetación natural de Chile. Santiago, Chile. Universitaria. $165 \mathrm{p}$.

Gaudin S. 1997. L'approche typologique et son utilité en foresterie. BTSA Gestion Forestière. Consultado 1 sept. 2009. Disponible en http://pagesperso-orange.fr/le.mago/Acrobat/ Typo.pdf

Gómez-Manzanedo P, S Roig, J Reque. 2008. Caracterización selvícola de los hayedos cantábricos: influencia de las condiciones de estación y los usos antrópicos. Investigación Agraria 2 (17): 155-167.

Herbert I, F Rebeirot. 1985. Les futaies jardinées du Haut-Jura. Revue forestière française 38(6) 465-481.

Kent M, P Coker. 1992. Vegetation description and analysis. A practical approach. London, England. John Wiley. 363 p.

Ministerio de Salud y Ambiente. 2005. Primer Inventario Nacional de bosques nativos. SAyDS. 126 p. Consultado 1 sept. 2009.
Disponible en: http://medioambiente.gov.ar/archivos/web/ UMSEF/File/PINBN/informe_nacional_pinbn.pdf

Luebert F, P Pliscoff. 2006. Sinopsis bioclimática y vegetacional de Chile. Santiago, Chile. Universitaria. 307 p.

Luis-Calabuig E, R Tárrega, L Calvo, L Valvuena, E Marcos. 2001. Fuego y paisaje en áreas de dominio de roble rebollo. Ecosistemas 10 (1). Consultado 20 ago. 2008. Disponible en http://www.revistaecosistemas.net/articulo.asp?Id=377

Madrigal A, J Álvarez, A Rojo, R Rodríguez. 2000. Tablas de producción para los montes españoles. Madrid, España. Fundación Conde del Valle de Salazar. 260 p.

McGarigal K, S Cushman, S Stafford. 2000. Multivariate Statistics for Wildlife and Ecology Research. New York, USA. Springer. 279 p.

Martín Q, M Cabero, R Ardanuy. 1999. Paquete estadístico spss 8.0. Salamanca, España. Hespérides. 412 p.

Pardo A, A Ruiz. 2005. SPSS 10. Guía para el análisis de datos. Madrid, España. Universidad Autónoma de Madrid. $569 \mathrm{p}$.

Piqué M, J Romà, P Vericat. 2007. Caracterización de las masas de robles submediterráneos (Quercus humilis e híbridos afines) como base para la definición de propuestas de gestión en el Pirineo Catalán. Cuad. Soc. Esp. Cienc. For. 21: 67-74.

Reque J. 2004. Estructura, dinámica y selvicultura de los bosques de roble albar (Quercus petraea Matts. Liebl.) en la Cordillera Cantábrica meridional. Tesis doctor. Palencia, España. Departamento de Producción Vegetal y Recursos Forestales, Universidad de Valladolid. 269 p.

Reque J, M Sarasola, M Gyenge, M Fernández. 2007. Caracterización silvícola de nirantales del norte de la Patagonia para la gestión forestal sostenible. Bosque 28 (1): 33-45.

Rivas-Martínez S. 1976. Sinfitosociología, una nueva metodología para el estudio del paisaje vegetal. Anales Inst. Bot. Cavanilles 33: 179-188.

Rivas-Martínez S, A Penas. 2003. Atlas y manual de los hábitats de España. Madrid, España. Dirección General de Conservación de la Naturaleza, Ministerio de Medio Ambiente. 492 p.

Rivera H. 2007. Ordenamento territorial de áreas florestais utilizando avaliação multicritério apoiada por geoprocessamento, fitossociologia e análise multivariada. Tesis Mestrado en Ciências Florestais. Curitiba, Brasil. Setor de Ciências Agrárias, Universidade Federal do Paraná. 225 p.

Roig S, P Alonso, M Río, G Montero. 2006. Tipología dasométrica de masas puras y mixtas de sabina albar (Juniperus thurifera L.) españolas. In Actas III Coloquio internacional sobre sabinares y enebrales (género Juniperus): ecología y gestión forestal sostenible. Soria. España. p. 177-185.

Roig S, M Del Río, R Ruiz-Peinado, I Cañellas. 2007. Tipología dasométrica de los rebollares (Quercus pyrenaica Milld.) de la zona centro de la Península Ibérica. In Actas XLVI Reunión científica de la SEEP. Vitoria, España. p. 535-542.

Santos L, P Herrera, M Ceballos, O Parrilla, A Martínez. 2005. Adaptación y revisión del PGOU de Ponferrada, León. Ponferrada, España. Diagnóstico Ambiental, Planteamiento Urbanístico S.I. 320 p.

Serrada R, I González, C López, B Marchal, A San Miguel, E Tolosana. 1993. Tipificación dasométrica de los rebollares (Quercus pyrenaica Willd.) de la comunidad de Madrid. In Alternativas silvopastorales. Diseño de un plan experi- 
mental. Congreso Forestal Español. Tomo II. Pontevedra, España. p. 623-629.

Tárrega R, L Calvo, E Marcos, A Taboada. 2006. Forest structure and understory diversity in Quercus pyrenaica communities with different human uses and disturbances. Forest Ecology and Management 227: 50-58.
Vila P, I Díaz-Maroto. 2002. Las masas actuales de Quercus petrea en Galicia. Investigaciones Agrarias 11 (1): 5-28.

Vita A. 1996. Los tratamientos silviculturales. Santiago, Chile. Escuela de Ingeniería Forestal, Universidad de Chile. 147 p.

Recibido: 03.09.08

Aceptado: 07.09.09 